\title{
Risks associated with the transportation of hazardous materials on public roads
}

\author{
Eligiusz Mieloszyk ${ }^{1}{ }^{*}$, and Anita Milewska ${ }^{1}$ \\ ${ }^{1}$ Gdańsk University of Technology, Gabriela Narutowicza 11/12, 80-233 Gdańsk, Poland
}

\begin{abstract}
A significant proportion of the transport of hazardous materials is carried out on public roads. Therefore, the safety of such transport is becoming increasingly important. Every catastrophe involving hazardous materials has a negative impact on direct road users and the surrounding environment, becauses its range is mostly not local. It follows that in the event of such catastrophe, its effects should be minimized. This is possible only when we know the mechanism of spreading effects of a catastrophe involving hazardous materials. Those effects are spread by two basic media: ground (in particular water in the ground) and air. Sometimes those effects are spread by water, while the catastrophe has occurred near a water reservoir or watercourse with a free flow surface. In extreme cases, this can even lead to an ecological disaster. Dynamic systems, especially those with distributed parameters, can be used to describe the mechanism of the disaster's spread. Properties of phenomena accompanying analyzed catastrophes are well reflected in their linear or non-linear mathematical models $[1,2]$, which are analyzed by various operator methods [3].
\end{abstract}

\section{Introduction}

The purpose of the article is not to deal with conditions which should be satisfied by the transport of dangerous goods, because those are settled by relevant regulations. Such transports must satisfy very strict safety requirements, referring to both drivers, vehicles and their equipment. However, risk associated with the transport of dangerous goods on public roads often occurs for various reasons. This is not only about the handling of dangerous chemicals, but also explosives, technical gases, materials that generate flammable gases in contact with water, etc.

These hazardous substances pose a threat to lives of people, animals and to the environment. Transporting large amounts of these materials is a problem. For example, large quantities of sulphuric acid $\mathrm{H}_{2} \mathrm{SO}_{4}$ are transported in tanks with a capacity of over $20 \mathrm{t}$. As a result of uncontrolled release of significant quantities of dangerous substances with oxidizing, flammable, toxic, etc. properties to the environment (leakage during a collision, unsealing of the tanker during transport, traffic accident, etc.) there may be a risk of ecological disaster, which is a serious threat to people and the environment.

\footnotetext{
*Corresponding author: eligiusz.mieloszyk@.pg.edu..pl
} 
Pollution/contamination occurring on the occasion of road collisions is generally local, but in selected cases their range may be much wider, because they can travel over long distances in the air (wind effect), in surface water, in groundwater or in the ground.

It should also be noted that on regular basis during the use of car tyres in typical road conditions noise is emitted, which is currently one of the most troublesome environmental problems and dust is generated during tread wear, and rolling resistance affects fuel consumption, and thus $\mathrm{CO}_{2}$ emissions. and other chemical compounds. For example, according to the Central Statistical Office, the total emission of air pollutants related to the consumption of tyres, brakes and road surfaces in Poland in 2011, amounted to about 63 thousand tonnes.

Disasters in road transport take place as a result of the so-called human factors, i.e. errors of the driver or other users of road traffic, improper securing of cargo (e.g. tanks before transport), etc. Second important group of factors that cause adverse events are technical failures of vehicles or of road infrastructure - Fig. 1.

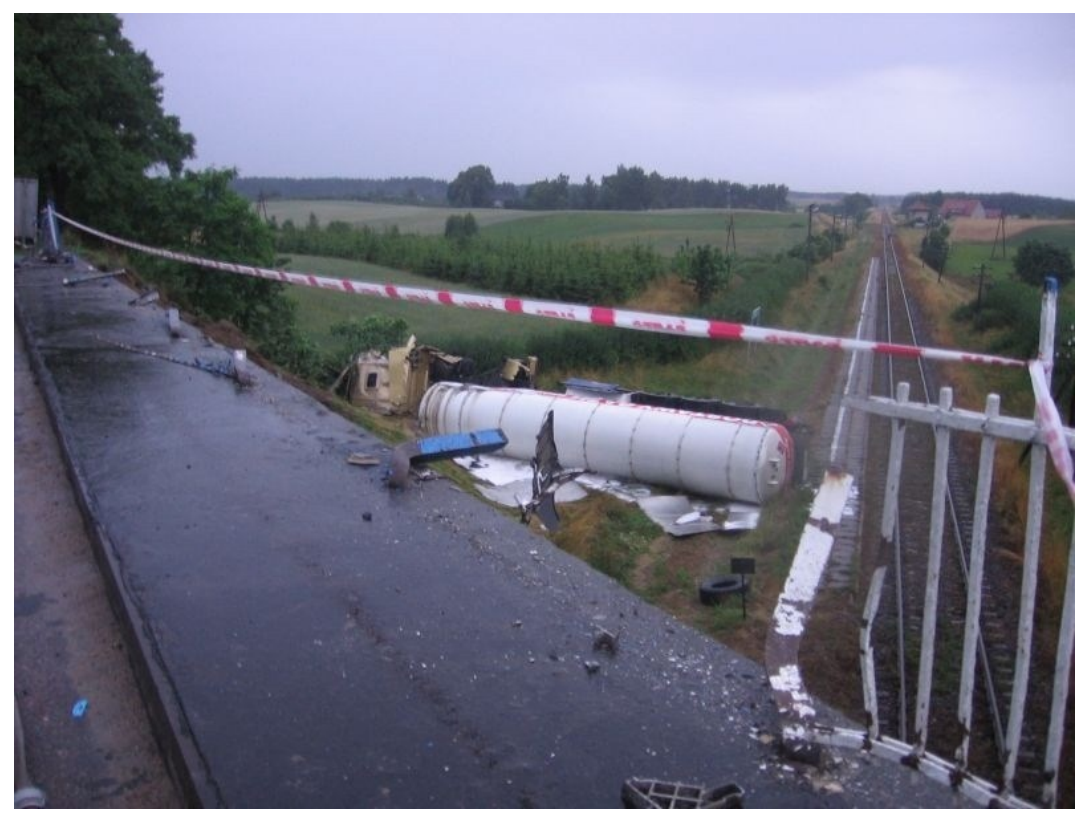

Fig. 1. Fall from the viaduct of the car - a cistern with 20t of propane-butane gas (source: Provincial Police Headquarters - Gdańsk).

Disaster is a dynamic process [2,3] which is particularly uncontrollable in the initial phase, so we should strive to make the transition to controlling this process as soon as possible, because only then can the consequences of the disaster be reduced. Disasters are generally accompanied by:

- leaking petroleum materials from tanks - no fire

- leaking petroleum materials from tanks connected with a fire

- leaking other hazardous materials (e.g. chlorine, sulphuric acid $\mathrm{H}_{2} \mathrm{SO}_{4}$ )

- the spread of dangerous volatile compounds

- other factors.

\section{Emission of disaster effects by air}

In each of these cases, there is also the emission of fumes that have negative impact on the respiratory tract and skin, especially of those who are in the area of the disaster, and mainly 
those taking part in the rescue operation. These are, among others, low-volatile components and volatile organic compounds, whose range of environmental impact and direct influence on the aerosanitary state is high, similar to the emission of bitumen fumes in road construction [4], and this emission in the case of volatile organic compounds amounts to $0.1-2.0 \mathrm{mg} / \mathrm{m} 3$ $-[5]$.

The use of multidimensional or non-linear models $[2,6]$ is often unnecessary, because even in the case of vertical diffusion (vertical pollution - vertical exchange of pollutants) it is enough to take into account the layered pollutant principle of utilization, as in [7], which uses the estimated amount of contaminants of the $i$-th layer of depth $h_{i}$, penetrating into the neighbouring layer. In the case of spreading pollutants in the atmosphere, the wind field approximation is necessary.

Particles of any medium (solid, liquid, gaseous) are in continuous accidental motion, the intensity of which is determined by the temperature of a given medium. As a result of this movement, molecules exchange between neighbouring layers of the medium, causing a transfer phenomenon called diffusion transfer, often called diffusion for short. If layers have different temperature, the exchange causes heat transfer. If they are moving with different average velocities, there is a transfer of the momentum. If we deal with a liquid medium with admixtures (e.g. with impurities), the difference in concentration of admixture in adjacent liquid layers (in our case mainly water) causes mass transfer. Values of individual magnitudes are directly proportional to appropriate spatial gradients of temperature or of concentration. Flow coefficients depend on properties of the medium, and their dependence on temperature, pressure and concentration is determined experimentally in most cases. The basic equation describing the transfer of thermal energy is the Fourier equation. Assuming one-dimensional flow, it takes the form analogous to the diffusion equation, i.e. in mathematical modeling they are described by analogous formulas using differential equations of the parabolic type. In the general case, the mathematical form of the pollution spreading model is a combination (parallel or chain) of the description of individual component processes, among which the diffusion process is important, which in its description is analogous to the description of thermal conductivity [1].

The one-dimensional homogeneous diffusion equation in the layered model for vertical diffusion looks as follows:

$$
\frac{\partial}{\partial \mathrm{z}}\left(K_{V_{i}} \frac{\partial u_{i}}{\partial \mathrm{z}}\right)=\frac{\partial u_{i}}{\partial \mathrm{t}}, \quad i=1,2,3
$$

where $K_{V_{i}}=$ const is the diffusion coefficient for the $i$-layer, $u_{i}$ is the concentration of the diffusible component in place $z$ at time $t$ in the $i$-layer.

The solution of equation (1) takes the form [8]

$$
u_{i}(z, t)=\frac{1}{\sqrt{4 \pi K_{V_{i}} t}} \exp \left(-\frac{z^{2}}{4 K_{V_{i}} t}\right)
$$

and shows the development of the phenomenon for a very "sudden" appearance of pollution accompanying disasters at time $t=0$.

The course of values $K_{V_{i}}(z)$ in the ground level can be approximated by the formula:

$$
K_{V_{i}}(z)=\frac{\mathrm{\kappa} u_{*} z}{\emptyset_{H}\left(\frac{Z}{L}\right)} \quad, \quad 0 \leq z \leq h
$$


where $\mathrm{K}$ is the von Karman constant, $u_{*}$ is the friction speed, $L$ is the Monina-Obukhov parameter, and $\emptyset_{H}$ is the universal function defined in [9].

The presented model (1) can be extended with the component responsible for advection, which is directly affected by the wind field. If there is given the set $Q \times$ $(0, T)$, where $Q=\Omega \times(0, H), \Omega \subset R^{2}$, H is the height of the hash layer, and $\mathrm{T}$ is the forecast time, then extended model can be written as follows:

$$
\frac{\partial u_{i}}{\partial t}+\vec{V} \cdot \nabla u_{i}=\nabla K \nabla u_{i}+R_{i}\left(u_{1}, u_{2}, \ldots, u_{n}\right)+S_{i} \quad i=1,2, \ldots, n .
$$

In last differential equation, following designations were adopted: $\vec{V}=[u, v, w]$ is a wind field vector, $K$ is a turbulent diffusion tensor (see also chapter 3 of this article), $S_{i}$ is the emission field from high sources, $R_{i}$ are the coefficients of physicochemical reactions. The influence of fields of emission from surface sources is taken into account in boundary conditions [10]. It is not possible to determine the response of a complex dynamic system (2). Only the adoption of simplifying assumptions makes it possible to carry out a detailed analysis of this dynamic system. Assuming in the model (2) the following simplifying assumptions: physico-chemical changes are omitted, the concentration field and basic atmospheric factors are unchanged in the assumed forecast range, atmospheric air in the region is treated as incompressible and the wind field generates horizontal wind consistent with the positive axis orientation $\mathrm{x}$. With these assumptions and with appropriate initialboundary conditions attached to the model, we can determine the response of this system. It assumes the following form:

$$
\begin{aligned}
u_{i}(x, y, z)= & \frac{Q}{2 \pi|u| \sigma_{h} \sigma_{z}} \exp \left(-\frac{y^{2}}{2 \sigma_{h}^{2}}\right)\left\{\exp \left(-\frac{(z-H)^{2}}{2 \sigma_{z}^{2}}\right)\right. \\
& \left.+\exp \left(-\frac{(z+H)^{2}}{2 \sigma_{z}^{2}}\right)\right\}
\end{aligned}
$$

where $u$ is the first component of the wind field according to the assumption, $Q$ is the point source emission value, $\sigma_{h}=A x^{a}, \sigma_{z}=B x^{b}$. In dependencies for $\sigma_{h} i \sigma_{z}$ parameters $A, B, a, b$ should be determined by empirical methods [11]. The presented model with accepted simplifications and its response works well on a local scale, and this is the case when effects of catastrophes involving hazardous materials are considered.

\section{Emission of effects of a catastrophe in the ground}

Pollution from disasters can dissolve in water or move with it as slurry. In the case of a disaster involving hazardous materials, it is important to model the propagation of groundwater pollution. Petroleum compounds leak into the ground not only in the event of a collision, but also as a result of accidental leakage during transport, handling and refueling, for example at petrol stations, etc. They contain toxic compounds such as benzene, toluene or xylenes. In unfavorable conditions, they can migrate over long distances, and the cleaning of groundwater is associated with high costs. The assessment of the impact of these and other pollutants on the quality of groundwater, and especially the design of measures to minimize negative effects of contamination requires the creation of models that will allow for qualitative and quantitative prediction of migration of pollutants in the ground. These migrations depend on hydrogeological properties of the soil. Pollutants that get into the ground flow down under the influence of gravity and due to the permeability of the medium 
they reach the underground water. This flow through the porous medium is described by Darcy's law - [12]

$$
q=-K \Delta \emptyset / L
$$

where $q$ is the flow of water through a section of the medium with a unit cross-sectional area and a volume $L$ in a unit of time at a hydraulic gradient $\Delta \emptyset . K$ is the hydraulic conductivity coefficient, and the minus sign means that the direction of flow is opposite to the direction of potential growth.

If $\vec{q}$ is a vector unit flow, then for an isotropic medium, i.e. a medium in which the permeability is not dependent on the direction, Darcy's law is:

$$
\vec{q}(\vec{x}, t)=-K(\vec{x}) \operatorname{grad} \emptyset(\vec{x}, t)
$$

For the anisotropic medium in which the permeability depends on the direction, the hydraulic conductivity is the tensor represented by the symmetrical matrix in the form:

$$
K=\left[\begin{array}{lll}
K_{x x} & K_{x y} & K_{x z} \\
K_{y x} & K_{y y} & K_{y z} \\
K_{z x} & K_{z y} & K_{z z}
\end{array}\right]
$$

which after entering appropriate coordinate system can be reduced to a diagonal form

$$
K=\left[\begin{array}{ccc}
K_{x} & 0 & 0 \\
0 & K_{y} & 0 \\
0 & 0 & K_{z}
\end{array}\right]
$$

If water density is constant, then the mass conservation equation [10] is as follows:

$$
-\operatorname{div} \vec{q}=\frac{1}{V_{0}} \frac{\partial V_{w}}{\partial t}
$$

If

$$
\frac{1}{V_{0}} \frac{\partial V_{w}}{\partial t}=\frac{1}{V_{0}} \frac{\partial V_{w}}{\partial \emptyset} \frac{\partial \emptyset}{\partial t},
$$

so using the elastic filtration $S_{0}$ which is defined by the formula

$$
S_{0}=\frac{1}{V_{0}} \frac{\partial V_{w}}{\partial \emptyset}
$$

where $V_{0} i V_{w}$ are the volume of the medium and the volume of water respectively, it can be recorded using the Darcy's equation:

$$
\operatorname{div}(K(\vec{x}) \operatorname{grad} \emptyset)=S_{0} \frac{\partial \emptyset}{\partial t}
$$

In last equation, there is a quantity $\emptyset$ whose determination allows to determine the unit flow and this is the basic equation describing the movement of water, which is the medium responsible for the spread of contaminations, pollution. 
If we accept the coordinate system so that the tensor $\mathrm{K}$ will be a diagonal tensor, then last equation will be simplified:

$$
\frac{\partial}{\partial x}\left(K_{x} \frac{\partial \emptyset}{\partial x}\right)+\frac{\partial}{\partial y}\left(K_{y} \frac{\partial \emptyset}{\partial y}\right)+\frac{\partial}{\partial z}\left(K_{z} \frac{\partial \emptyset}{\partial z}\right)=S_{0} \frac{\partial \emptyset}{\partial t}
$$

In the case of a homogeneous, isotropic medium, last differential equation (5) will take the form:

$$
K\left(\frac{\partial^{2} \emptyset}{\partial x^{2}}+\frac{\partial^{2} \emptyset}{\partial y^{2}}+\frac{\partial^{2} \emptyset}{\partial z^{2}}\right)=S_{0} \frac{\partial \emptyset}{\partial t}, K=K_{x}=K_{y}=K_{z}
$$

If the flow is stationary, then for $\emptyset$ we get the Laplace equation in the form:

$$
\frac{\partial^{2} \emptyset}{\partial x^{2}}+\frac{\partial^{2} \emptyset}{\partial y^{2}}+\frac{\partial^{2} \emptyset}{\partial z^{2}}=0
$$

If the pollution changes the water density, i.e. $\rho=\rho(c)$, where $\mathrm{c}$ is the concentration of the contaminant then the mass conservation equation will take the form:

$$
-\operatorname{div} \rho \vec{q}=\frac{1}{V_{0}} \frac{\partial\left(\rho V_{w}\right)}{\partial t}
$$

Let's calculate $\frac{\partial\left(\rho V_{w}\right)}{\partial t}$ and use the formula $\operatorname{div} \rho \vec{q}=\vec{q} \operatorname{grad} \rho+\rho \operatorname{div} \vec{q}$ of field theory.

Then we get

$$
\frac{\partial\left(\rho V_{w}\right)}{\partial t}=V_{w} \frac{\partial \rho}{\partial t}+\rho \frac{\partial V_{w}}{\partial t}
$$

so:

$$
\frac{1}{V_{0}} \frac{\partial\left(\rho V_{w}\right)}{\partial t}=\frac{V_{W}}{V_{0}} \frac{\partial \rho}{\partial t}+\frac{\rho}{V_{0}} \frac{\partial V_{w}}{\partial t}=-\vec{q} \operatorname{grad} \rho-\rho \operatorname{div} \vec{q}
$$

in other words:

$$
-\vec{q} \operatorname{grad} \rho-\rho \operatorname{div} \vec{q}=\frac{V_{W}}{V_{0}} \frac{\partial \rho}{\partial t}+\rho S_{0} \frac{\partial \emptyset}{\partial t}
$$

Assuming that $P=\frac{V_{w}}{V_{0}}$ is the porosity of the center, last dependence can be written in the form:

$$
-\vec{q} \operatorname{grad} \rho-\rho \operatorname{div} \vec{q}=P \frac{\partial \rho}{\partial t}+\rho S_{0} \frac{\partial \emptyset}{\partial t}
$$

With small changes in density, it can be assumed that first components on both sides of last equality are small enough and can be omitted. After taking this fact into account, in this case we will also get the equation of form (4). This means that in the next model we get the 
same equation describing given problem. From this it follows that the spread of dangerous compounds in the ground caused by a road accident can generally be described by the equation (4) and used in practice to limit its negative effects.

As was already mentioned, a road accident involving hazardous materials may be accompanied by fire. It is additionally dangerous if, for example, a gas pipeline runs nearby. Then such an uncontrolled rise in the temperature of the ground may additionally lead to gas explosion and cause further losses. In this case, it is important to describe vertical changes of temperature in the ground. They can be determined from the equation:

$$
\frac{\partial(c T)}{\partial t}=\frac{\partial}{\partial z}\left(K \frac{\partial T}{\partial z}\right)
$$

where $T$ is the temperature, $c$ is the thermal capacity of the unit of ground volume, and $K$ is the thermal conductivity of the ground. Using this equation, we can determine the temperature distribution depending on time and depth, and by attaching to it the component $f(z, t)$ characterizing the temperature source, we can additionally take into account the influence of the heat emission source.

\section{Summary}

The quantity of transports of dangerous goods by roads continues to grow. As a result of disasters involving hazardous materials, we may have to face for example, fires or contamination of air, soil and water. Disasters involving hazardous materials cause many tragedies, fatal poisoning, burns, etc. They have a negative impact on the environment.

In the event of a disaster, its negative effects should be minimized. Knowledge of properties and course of the phenomena accompanying road accidents is helpful in limiting effects of these accidents. Properties of phenomena accompanying the analyzed catastrophes are well reflected in their mathematical models, which are full of analogies between the phenomena of air, water and ground flows, including the flow of heat. These analogies can be further referred to electric current and always analyzed using operator methods. Used operator calculus methods simplify the notation of presented models and facilitate their analysis and simplify the assumptions. The phenomena accompanying analyzed disasters can be studied using analogue models and applied in practice to reduce effects of disasters.

\section{References}

1. L.C. Evans, Partial differential equations (American Mathematical Society, 2002)

2. A. Milewska, A solution of non-linear differential problem with application to selected geotechnical problems, Arch. Civ. Eng. LVII(2) pp. 187-197 (2011)

3. E. Mieloszyk, Non-classical operational calculus in application to generalized dynamical systems (in Polish) (Polish Academy of Sciences Scientific Publishers, Gdansk, 2008)

4. G.D. Roskam, R.N.J. Comans, Availability and leaching of polycyclic aromatic hydrocarbons: Controlling processes and comparison of testing methods, Waste Manag. 29 pp. 136-142 (2009). doi:10.1016/j.wasman.2008.03.013

5. I. Burstyn, H. Kromhout, C. Johansen, S. Langard, T. Kauppinen, J. Shaham, G. Ferro, P. Boffetta, Bladder cancer incidence and exposure to polycyclic aromatic hydrocarbons among asphalt pavers, Occup. Environ. Med. 64 pp. 520-526 (2007). doi:10.1136/oem.2006.029801 
6. R. Edwards, Functional analysis. Theory and applications (New York, 1965)

7. P. Holnicki, A. Kałuszko, A. Żochowski, A microcomputer implementation of air quality forecasting system for urban scale, Microcomput. Appl. 13(2) pp. 76-84 (1994)

8. E. Mieloszyk, S. Grulkowski, A. Milewska, The propagate of the effects of large railway disasters (in Polish), Arch. Inst. Civ. Eng. 25 pp. 301-310 (2017)

9. S.R. Hanna, G.A. Briggs, R.P. Hosker, Handbook of Atmospheric Diffusion (Technical Information Center, U. S. Department of Energy, 1982)

10. P. Holnicki, Z. Nahorski, A. Żochowski, Modeling of environmental processes (in Polish) (Publisher of the College of Applied Computer Science and Management, Warsaw, 2000)

11. M. Nowicki, W. Jaworski, Designing of industrial plants in the aspect of atmosphere protection (in Polish) (Warsaw University of Technology Publishers, Warsaw, 1986)

12. P.S. Eagleson, Dynamic hydrology (in Polish) (Polish Scientific Publisher, Warsaw, 1978) 